\section{Todesursachen beim Hyper-IgE-Syndrom}

\author{
Das Hyper-IgE- oder Hiob-Syndrom ist eine sehr seltene Form der \\ Immundefizienz. Bislang ist nur sehr wenig darüber bekannt, an \\ welchen Komplikationen die betroffenen Patienten letztlich sterben.
}

B eim Hyper-IgE-Syndrom (HIES) fallen nicht nur sehr hohe IgE-Konzentrationen, rekurrierende Infekte und Ekzeme auf, sondern auch zahlreiche andere Symptome wie Skoliose, Osteopenie, Dentitionsstörungen oder Kraniosynostosen. Die Störungen des Immunsystems sind nicht einheitlich und können die humorale sowie die zelluläre Abwehr betreffen. Typische Komplikationen beim HIES sind rezidivierende Pneumonien, häufig durch Staphylococcus aureus ausgelöst. Im weiteren Verlauf bilden sich häufig charakteristische Pneumatozelen, bei denen Pilze oder gramnegative Bakterien zu Infektionen führen.

Ein US-amerikanisches Forscherteam wertete retrospektiv die Daten von sechs Patienten mit der Diagnose HIES aus, die im National Institute of Health in Bethesda, Maryland, autopsiert wurden. Alle Patienten waren weiblich und verstarben im Alter zwischen 24 und 40
Jahren. Die jeweils höchsten IgE-Werte reichten von 12.800 bis $38.572 \mathrm{IU} / \mathrm{ml}$. Neben Ekzemen waren bei allen Patientinnen vor allem Pneumonien bekannt, mit einer Erstmanifestation bereits im Kindesalter. Alle wiesen zudem Lungenzysten auf. Sämtliche Frauen hatten zum Todeszeitpunkt eine Pneumonie mit Pseudomonas aeruginosa oder Pilzspezies, meist Aspergillus.

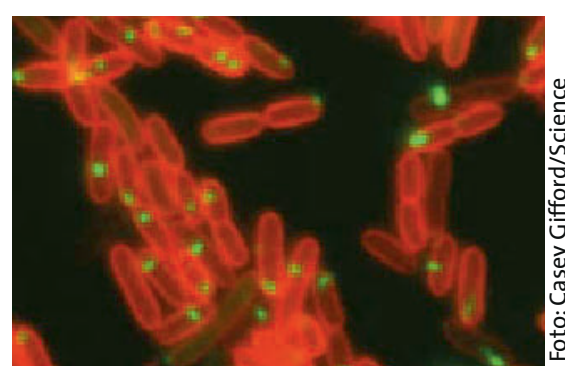

Pulmonale Infektionen mit Pseudomonas aeruginosa markieren oft die finale Phase bei Patienten mit Hyper-IgE-Syndrom.
Drei Patientinnen verstarben plötzlich außerhalb einer Klinik, Todesursache war jeweils eine bronchopulmonale Infektion. Zwei davon starben aufgrund einer akuten massiven pulmonalen Blutung. Bei den drei anderen Patientinnen war ein längerer stationärer Verlauf vorangegangen, der trotz intensiver antimikrobieller Therapie zum Exitus führte. Eine Patientin verstarb an respiratorischer Insuffizienz, bei zwei führten zerebrale mykotische Herde zum Tode. Bei vier der sechs Patientinnen zeigten sich renale tubuläre Nierenschäden, die möglicherweise auf die Toxizität von Amphotericin B zurückzuführen waren. Drei der Verstorbenen wiesen eine Glomerulosklerose auf.

Fazit: Patienten mit HIES verstarben nach dieser Untersuchung an pulmonalen oder zerebralen Infekten durch Pseudomonas- oder Aspergillus-Spezies. Ein optimale antimikrobielle Prophylaxe und Therapie ist für diese Patienten essenziell.

Freeman AF et al. Causes of death in hyper-IgE syndrome. J Allergy Clin Immunol 2007; 119: 1234-40

\section{Mehr Lebensqualität für Urtikariapatienten}

\author{
Eine idiopathische Urtikaria kann Lebensqualität und auch Arbeits- \\ leistung der Betroffenen deutlich einschränken. Ob ein Anti- \\ histaminikum diese Beeinträchtigung verringern kann, untersuchte \\ eine US-amerikanische Doppelblindstudie.
}

\footnotetext{
A llergologen aus den US-Bundesstaaten Kalifornien und Maryland rekrutierten für ihre multizentrische Studie 254 Patienten über zwölf Jahren mit chronischer idiopathischer Urtikaria. Nach einer zwei bis fünfTage dauernden Plazebogabe erhielten die Studienteilnehmer über 28 Tage randomisiert entweder einmal täglich 180 mg Fexofenadin- $\mathrm{HCl}(\mathrm{n}=163)$ oder Plazebo ( $\mathrm{n}=$ 91). Primäre Endpunkte der Studie waren Quaddelbildung und Juckreiz, die durch das Antihistaminikum im Vergleich zu Plazebo signifikant verringert wurden. Diese Ergebnisse sind bereits 2005 publiziert worden. In der aktuellen
}

Auswertung ging es jetzt um die Daten zur Lebensqualität und Produktivität. Zur Erfassung kamen die Fragebögen „Dermatology Life Quality Index“ (DLQI) und "Work Productivity and Activity Impairment" (WPAI) zu Beginn der Untersuchung sowie nach zwei und vier Wochen zum Einsatz.

Die Lebensqualität der Patienten unter Verum verbesserte sich über vier Wochen hinweg im Vergleich zur Plazebogruppe signifikant stärker $(\mathrm{p}=0,0219)$. Dies betraf insbesondere die DLQITeilskalen „Symptome und Emotionen“ $(p=0,0119)$ sowie „persönliche Beziehungen“ ( $p=0,0091)$. Auch die Produk- tivität war unter der Fexofenadinbehandlung signifikant besser als unter Plazebo: Die Beeinträchtigung bei der Arbeit durch die Urtikaria war deutlich geringer und die Aktivitäten waren weniger eingeschränkt. Die Überlegenheit von Fexofenadin über Plazebo zeigte sich sogar schon nach zwei Wochen. Zu diesem Zeitpunkt waren bereits vier von sechs DLQI- und zwei von vier WPAI-Teilaspekten signifikant besser als in der Kontrollgruppe.

Fazit: Bei chronischer idiopathischer Urtikaria verbessert die einmal tägliche Einnahme von $180 \mathrm{mg}$ Fexofenadin- $\mathrm{HCl}$ nicht nur die Symptomatik, sondern auch die Lebensqualität und die Arbeitsleistung der Betroffenen signifikant. $\quad f_{k}$

Spector SL et al. The effect of fexofenadine hydrochloride on productivity and quality of life in patients with chronic idiopathic urticaria. Cutis 2007; 79: 157-162 